\title{
Facile preparation of rich $\beta$-transcrystallinity in PET fiber/iPP composites
}

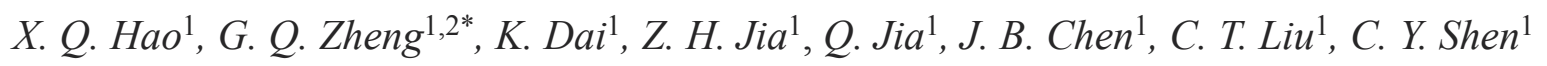 \\ ${ }^{1}$ College of Materials Science and Engineering, The Key Laboratory of Material Processing and Mold of Ministry of \\ Education, Zhengzhou University, Zhengzhou, 450002, P.R. China \\ ${ }^{2}$ The Key Laboratory of Polymer Processing Engineering, Ministry of Education, Guangzhou, 510641, P.R. China
}

Received 16 February 2011; accepted in revised form 7 June 2011

\begin{abstract}
Polyethylene terephthalate (PET) fibers containing different contents of $\beta$-nucleating agent ( $\beta$-NA) were meltspun from a reconstructive melt flow index rheometer at $270^{\circ} \mathrm{C}$, and then blended with polypropylene (iPP). The supermolecular and phase structure of the composite were investigated by polarized optical microscopy (POM), differential scanning calorimetry (DSC), and wide-angle X-ray diffraction (WAXD). For the pure PET fiber reinforced iPP, the interfacial region was mainly composed of $\alpha$-modification, though it has been demonstrated that PET fiber shows weak nucleating ability towards iPP matrix. More interestingly, when the amount of $\beta$-NA introduced into the PET fiber exceeds a critical value, the transcrystalline layer will be notably dominated by $\beta$-modification. This method to prepare $\beta$-transcrystallinity is remarkably different from those through stress or temperature control. The present results provide a facile and promising technique to prepare rich $\beta$-transcrystallinity under stress-free conditions.
\end{abstract}

Keywords: polymer composites, PET fiber, $\beta$-transcrystallinity, dendritic structure, self-organization

\section{Introduction}

It is well known that when fibers are introduced into a semi-crystalline polymer matrix, the matrix may be nucleated and fascinating crystalline morphology with various features will be developed at the interface. Such distinct morphology is defined as transcrystalline layer or transcrystallinity. This structure is always considered as an effective and economical method to improve interfacial adhesion [1-5].

Owing to the outstanding properties of $\beta$-iPP (e.g. better impact resistance and toughness) [6-11], $\beta$ crystallinty has attracted much attention in the past decades. Among them, $\beta$-transcrystallinity of iPP reinforced by fibers is particularly interesting. However, as for fiber/iPP composites, a number of studies have demonstrated that their interfacial morphology is mainly composed of $\alpha$-transcrys- tallinity. Up to now, a few special approaches such as pulling fiber in iPP melt [12-14] and controlling the fiber's introduction temperature [15-17], have been proposed to prepare $\beta$-transcrystallinity. Recently, $\beta$-NA was even chemically supported onto the surface of multi-wall carbon nanotubes by Wang et al. [18]. The results indicate that the multiwall carbon nanotubes after being supported with $\beta$ NA show enhanced $\beta$-nucleating ability towards iPP matrix. Except for the methods mentioned above, few approaches have been reported by which the $\beta$ transcrystallinity in fiber/iPP composites can be directly formed under static conditions.

Rare earth $\beta$-NA (WBG- II) exhibits considerable $\beta$-selectivity and nucleating efficiency to induce $\beta$ iPP $[19,20]$. There exist two crucial problems about the addition of $\beta$-NA: first, for directly mixed $\beta$ NA/iPP system, comparatively large amount of $\beta$ -

\footnotetext{
${ }^{*}$ Corresponding author, e-mail: gqzheng@zzu.edu.cn
} (c) BME-PT 
NA is needed; the second is that, owing to the addition of mass $\beta$-NA, the polymer composite's processability would be weakened. In view of these, a problem naturally arises: how to develop abundant $\beta$-modification on the premise that the extremely small content of $\beta$-NA is added. However, to the best of our knowledge, there are scarcely studies concerning: (1) rich $\beta$-transcrystallinity developed directly in PET fiber/iPP system under static condition and (2) the effect of the fraction of additive introduced into PET fibers on the crystalline modifications at the interface.

Owing to its scientific interest and industrial significance [21], in this study, we will focus on a facile method by which rich $\beta$-transcrystallinity can be created under quiescent condition. Inspired by work of Dai et al. [22], $\beta$-NA with different contents was first melt mixed with PET pellets, and then PET fiber containing $\beta$-NA was prepared via melt spinning. The spun PET fibers were introduced into iPP melt to get PET fiber/iPP composites. The crystalline morphology at the polymer composites' interface was investigated in detail by POM, DSC as well as WAXD. This study could provide an effective way to fabricate rich $\beta$-transcrystallinity in PET/ iPP composites in the absence of stress and temperature control.

\section{Experimental}

\subsection{Materials}

The iPP (T30S) used in this work is a commercial product from Lanzhou Petroleum Chemical Co., China. Its melt flow rate (MFR) is $2.6 \mathrm{~g} / 10 \mathrm{~min}$ $\left(230^{\circ} \mathrm{C}, 2.16 \mathrm{~kg}\right)$, and is ca. $11.0 \cdot 10^{4} \mathrm{~g} / \mathrm{mol}$. The PET pellet, which is blow molding grade with an intrinsic viscosity of $0.65 \mathrm{dl} / \mathrm{g}$, was purchased from Liaoyang Petroleum Chemical Co., China.

The $\beta$-NA (WGB-II), a heteronuclear bimetal complex of lanthanum and calcium with some specific ligands, was kindly provided by Winner Functional Materials Co. Ltd., China. It is an organic complex synthesized by rare earth elements and organic compounds (other information about this $\beta$-NA is available in the Supplement Material) [23]. Note that this kind of $\beta$-NA exhibits considerably $\beta$ selectivity and efficiency.

\subsection{Sample preparation}

To achieve a good dispersion of $\beta$-NA, WGB-II with different content $(0.5,1,1.5,2 \mathrm{wt} \%)$ was mixed with PET pellets in a Haake internal mixer (Haake Polylab System-Rheomex 252p series, RC9000, Haake company, Germany) at $270^{\circ} \mathrm{C}$, for 5 min. Pure PET pellets were also treated under the same conditions. After crushing and drying, the fibers containing $\beta$-NA (fibers' diameter is about $40 \mu \mathrm{m}$ ) were melt-spun by a reconstructive melt flow index rheometer (RL-5, Shanghai S.R.D. Scientific Instrument Company, Shanghai, China) at $270^{\circ} \mathrm{C}$ with a take-up speed of $3 \mathrm{~m} / \mathrm{min}$. In a convenient manner, the as-spun PET fiber is labeled as $\mathrm{x}$-PET, where $\mathrm{x}$ represents the mass percentage of $\beta$-NA. Hereafter, its corresponding composite is labeled as $\mathrm{x}-\mathrm{PET} / \mathrm{iPP}$. The schematic illustration of PET fiber preparation is shown in Figure 1.

PET fiber/iPP composites were prepared as follows: for the POM experiment, a given amount of iPP was put on a coverslip of a Linkam TMS 600 hot stage with a preset temperature of $200^{\circ} \mathrm{C}$, and then a single PET fibe was introduced into the iPP melt (Figure 1). Subsequently, another coverslip was put on and a film with a thickness of about $20 \mu \mathrm{m}$ was formed under the press of tweezers; For DSC and WAXD experiments, $5 \mathrm{wt} \%$ PET fibers and $95 \mathrm{wt} \%$ iPP were mixed in a Haake internal mixer (Haake Polylab System-Rheomex 252p series, RC9000, Haake company, Germany) at $200^{\circ} \mathrm{C}$, for $5 \mathrm{~min}$. The sample preparing procedure is shown in Figure 1. It is noteworthy that, before iPP was melt blended with PET fibers, PET fibers had been cut into short fibers with a length of ca. $5 \mathrm{~mm}$. In addition, the selection of $5 \mathrm{wt} . \%$ PET fibers in the composites has an important purpose: because of a smaller amount of $\beta$-transcrystallinity existing in single fiber reinforced composites, $\beta$-transcrystallinity might not be detected by WAXD and DSC. Therefore, $5 \mathrm{wt} \%$ PET fibers were introduced into iPP matrix to fabricate abundant $\beta$-transcrystallinity which can be detected easily by DSC and WAXD.

\subsection{Characterization}

\subsubsection{POM}

The polymer composites were held at $200^{\circ} \mathrm{C}$ for 5 min on a Linkam hot stage (TMS600) to remove 
the stress involved during sample preparation, and then cooled to room temperature at a cooling rate of $10^{\circ} \mathrm{C} / \mathrm{min}$. The morphological observation during cooling was carried out with a POM (Olympus BX61, Olympus Corporation, Japan). The fabrication procedure of the sample is illustrated in Figure 1(a composites).

\subsubsection{DSC}

Thermal analysis was performed on a DSC (TA DSC-2920, TA Instruments, USA). About 5 10 mg composite was sealed into an aluminum pan, heated from room temperature to $200^{\circ} \mathrm{C}$ at a heating rate of $10^{\circ} \mathrm{C} / \mathrm{min}$ and held for $5 \mathrm{~min}$ in order to erase the effect of thermal and mechanical history. Since $\beta$ iPP samples cooled down below $T(\alpha \beta)=100^{\circ} \mathrm{C}$ can recrystallize into the $\alpha$-modification during the partial melting of the $\alpha$-phase, the end temperature of cooling $\left(T_{\mathrm{R}}\right)$ was set at $100^{\circ} \mathrm{C}$. This limited recooling technique prevents $\beta \alpha$-recrystallisation $[11,24$, 25]. Then the samples were cooled to $100^{\circ} \mathrm{C}$ at a rate of $10^{\circ} \mathrm{C} / \mathrm{min}$. After that, they were heated to $200^{\circ} \mathrm{C}$ at a heating rate of $10^{\circ} \mathrm{C} / \mathrm{min}$. The DSC experiment has the same thermal process as POM.

\subsubsection{WAXD}

WAXD measurements were carried out on a Philips X'Pert Graphics and Indentify instrument (X'Pert PRO, PANalytical B.V., Netherlands) operating at $50 \mathrm{kV}$ and $30 \mathrm{~mA}$ with Ni-filtered $\mathrm{Cu} \mathrm{K}_{\alpha}$ radiation source. The composite film for WAXD test composed $5 \mathrm{wt} \%$ PET fibers and $95 \mathrm{wt} \%$ iPP and its

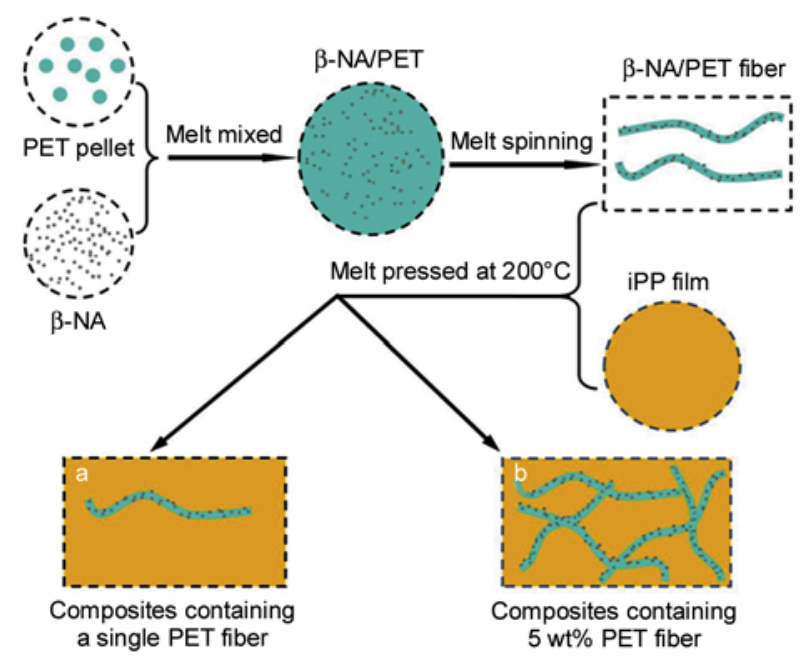

Figure 1. Schematic illustration of the preparation of PET fiber/iPP composites for characterization preparing procedure is also displayed in Figure 1(b composites). Note that it was thermally treated under the same conditions as those for POM and DSC measurements. Clearly, although the proportion of $\beta$-NA in PET fibers is between $0-2 \mathrm{wt} \%$, but the proportion of PET fibers in the composite is just $5 \mathrm{wt} \%$, so the proportion of $\beta$-NA in the composite is just $0-0.1 \mathrm{wt} \%$, which is a much smaller than that of iPP.

\subsubsection{Scanning electron microscope (SEM)}

The surface morphology of PET fiber was observed by a scanning electron microscopy (SEM, Quonxe2000, Phillips, FEI company, Netherlands) operating with an acceleration voltage of $20 \mathrm{kV}$. All the samples were sputtered with gold before taking images.

\section{Results and discussion \\ 3.1. The effect of $\beta$-NA loaded in PET fiber on the interfacial morphology}

The formation of the transcrystalline layer, originated by fiber surface, is generally studied by single fiber model composites. In this study, the samples were crystallized under non-isothermal conditions after the erasure of thermal and mechanical history. Figure 2a shows a series of optical micrographs for 0-PET/iPP taken at three representative crystallization stages (i.e., the beginning, medium, and the end) during the cooling process. It can be clearly seen that once the temperature decreases to ca. $128^{\circ} \mathrm{C}$, dense transcrystallinity immediately appears on PET fibers' surface. Simultaneously, dot-like nuclei are developed in the iPP matrix. With further decreasing temperature, the crystallites grow from these nuclei until they impinge on each other and a well developed transcrystalline layer can be obtained along the PET fibers. According to the birefringence of the crystals, it suggested that the crystallites in the matrix and those on the surface of PET fiber are of the same crystalline form. For the sample in which smaller amount of $\beta$-NA was added (0.5-PET/iPP), its matrix and interfacial feature is strongly analogous to that of 0 -PET/iPP (see Figure $2 b$ ), indicating that low content of $\beta$-NA loaded in PET fiber is not enough to change the interfacial morphology. Furthermore, this also confirmed that the PET fiber used in this study shows obvious heterogeneous nucleating ability toward iPP. 
Figure 2c-e shows the series of optical micrographs of 1-PET/iPP, 1.5-PET/iPP and 2-PET/iPP, respectively. It is very interesting that when the samples were cooled down to ca. $135^{\circ} \mathrm{C}$, many characteristic dendritic structures first appeared randomly on the lateral surface of PET fibers (see Figure 2c-e), and
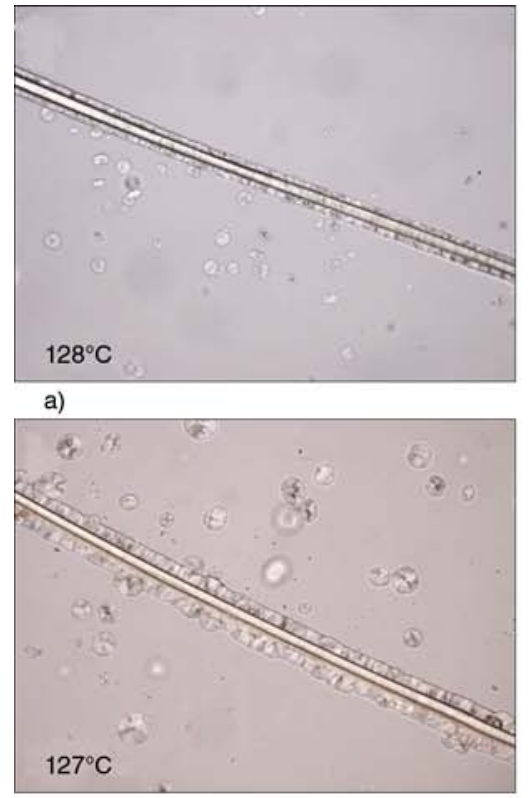

b)

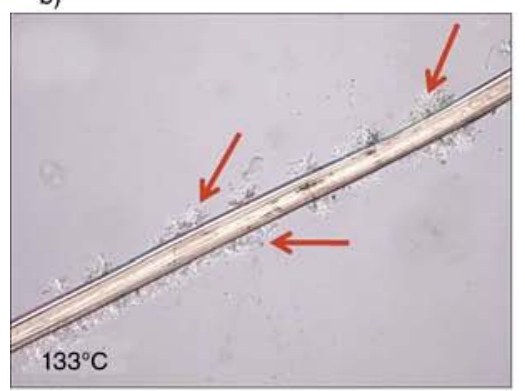

c)

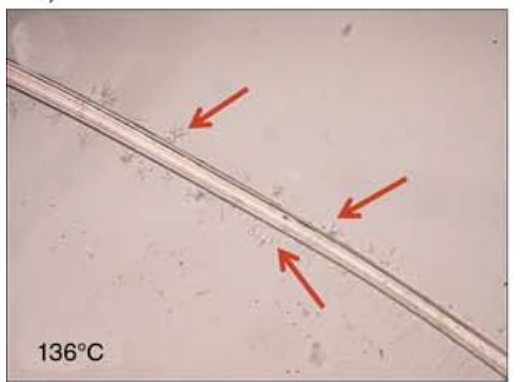

d)

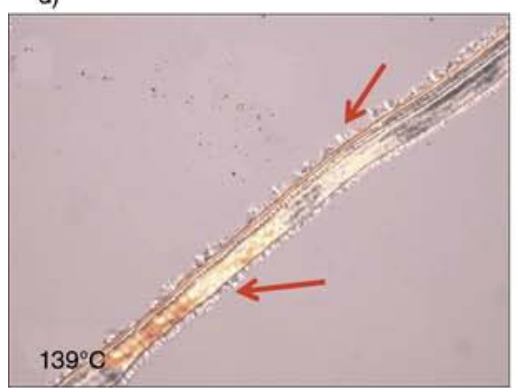

e)

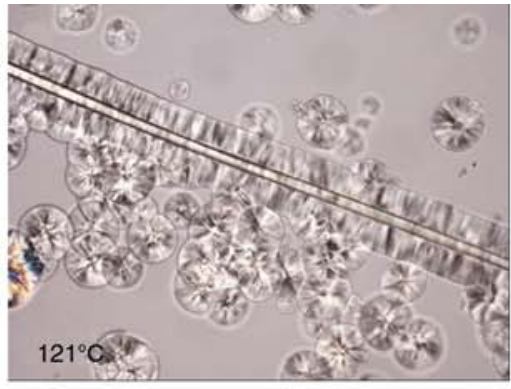

a')

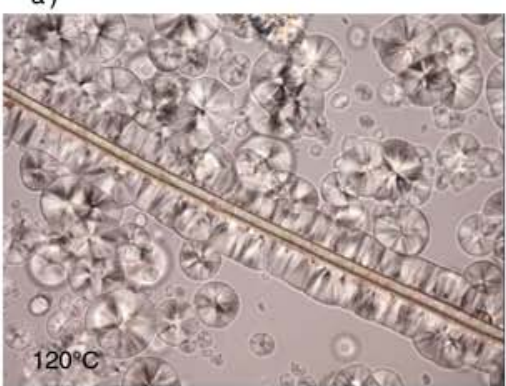

b')

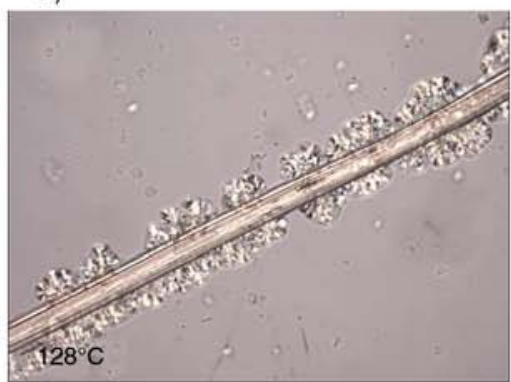

c')

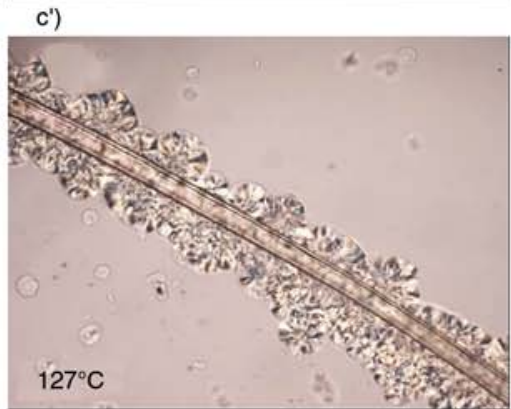

d')

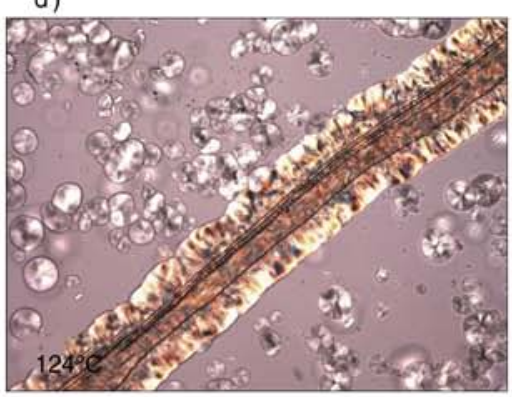

e')

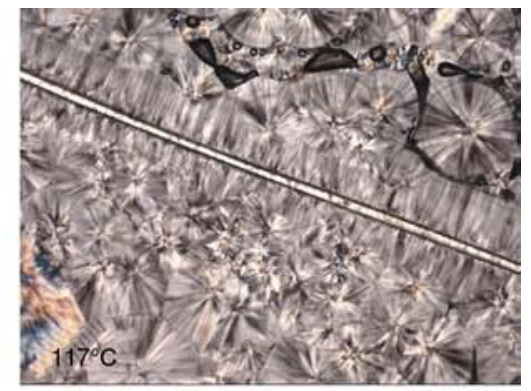

a")

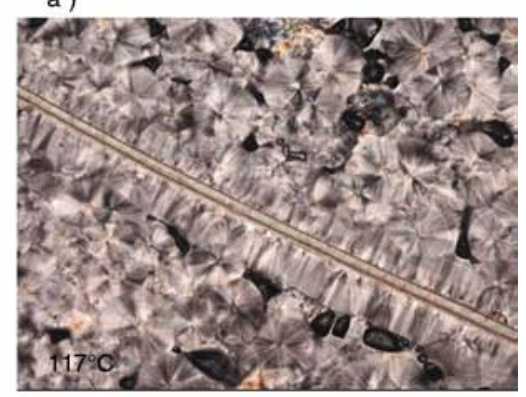

b")

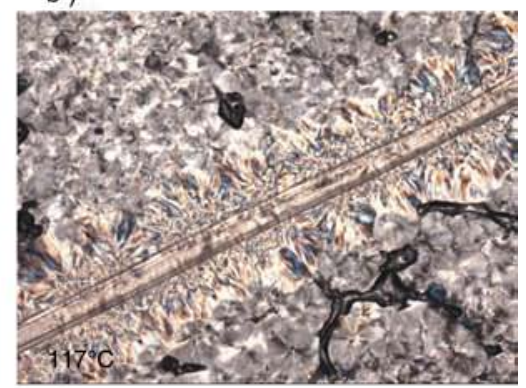

c")

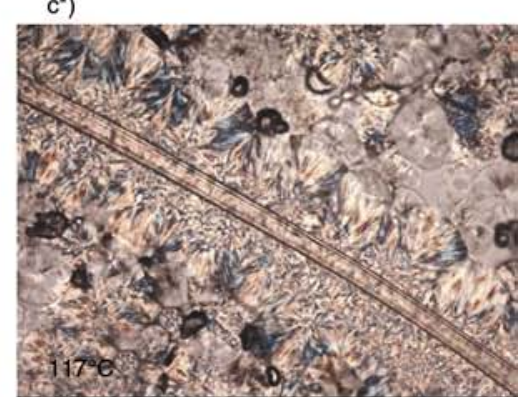

d")

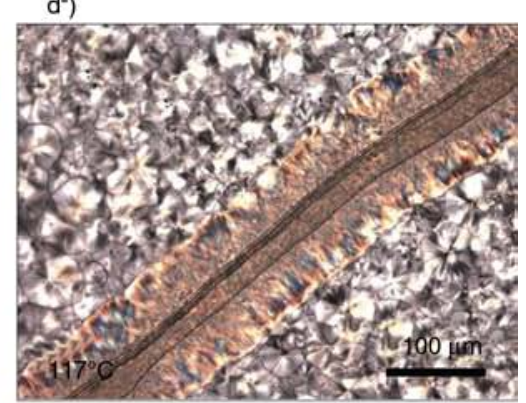

e)

Figure 2. A series of optical micrographs of the PET fiber/iPP composites taken at different temperatures during the cooling process: (a) 0-PET/iPP; (b) 0.5-PET/iPP; (c) 1-PET/iPP; (d) 1.5-PET/iPP; (e) 2-PET/iPP. Temperature decreases from the left image to the right one. 
the dendritic structures grew quickly with decreasing temperature. Compared with the crystals that emerged surrounding the PET fiber in 0-PET/iPP and 0.5-PET/iPP, the temperature at which the dendritic structures appeared is higher than that for 0 PET/iPP and 0.5-PET/iPP systems. Varga and Menyhárd [9] has also found the similar structure when they study the solubility and nucleating duality of N, N'-Dicyclohexyl-2, 6-naphthalenedicarboxamide (NJS) in iPP matrix. The formation of dendritic structures can be explained as follows: the $\beta$-NA can be partially molten at higher temperature, and then dendritic structures (i.e. nuclei) can be developed through high level of self-organization during cooling process.

Furthermore, as the temperature further decreases to $128^{\circ} \mathrm{C}$, nuclei in bulk begin to appear sporadically. Thus taking into account above results, the $\beta$ NA filled PET fiber shows strong heterogeneous nucleating ability toward iPP at the interface of the composite. Moreover, when the samples' temperature further decreased to $123^{\circ} \mathrm{C}$, the closely packed dendritic structures around the PET fiber caused the formation of transcrystallinity due to the limitation from the neighboring crystallites. Simultaneously, the bulk is still not fully occupied by the growing spherulites in matrix. This crystallization process is not completed until the sample is cooled to $116^{\circ} \mathrm{C}$. It is worth noting that the spherulites in the bulk and the transcrystallinity are not of the same birefrin- gence, suggesting that they are of different crystalline modifications.

To clarify what kind of crystalline forms are present in both the bulk and transtrystalline layers, these completely crystallized samples were heated to $158^{\circ} \mathrm{C}$ (this temperature is above the melting point of $\beta$-iPP but below that of $\alpha$-iPP) at a rate of $10^{\circ} \mathrm{C} / \mathrm{min}$. Figure 3 shows the optical micrographs of the composites after selective melting at $158^{\circ} \mathrm{C}$. In Figure $3 a$ and $b$, it is evident that neither the spherulites nor the transcrystalline layer in 0-PET/ iPP and 0.5-PET/iPP exhibit any change. More interestingly, as shown in Figure 3c-e, transcrystallinity in 1-PET/iPP, 1.5-PET/iPP and 2-PET/iPP significantly disappeared, resulting in the occurrence of sporadic fragmentary nuclei in the area near the surface of the PET fiber (shown by the arrows). Additionally, the spherulites in the matrix and the dendrites on the fibers' surface still exist. The selective melting test preliminarily confirms that the crystalline column surrounding the PET fiber in 0-PET/ iPP and 0.5-PET/iPP is $\alpha$-transcrystallinity, while that for $1-\mathrm{PET} / \mathrm{iPP}, 1.5-\mathrm{PET} / \mathrm{iPP}$ and $2-\mathrm{PET} / \mathrm{iPP}$ is $\beta$ transcrystallinity. During the crystallization process, the $\beta$-iPP grew faster and thus hindered the expansion of $\alpha$ front in the later stage of the crystallization. As a consequence, the transcrystallization formed in 1-PET/iPP, 1.5-PET/iPP and 2-PET/iPP were all $\beta$-modification. Dendrites have mixed polymorphic composite can be proved by presence of $\alpha$ -

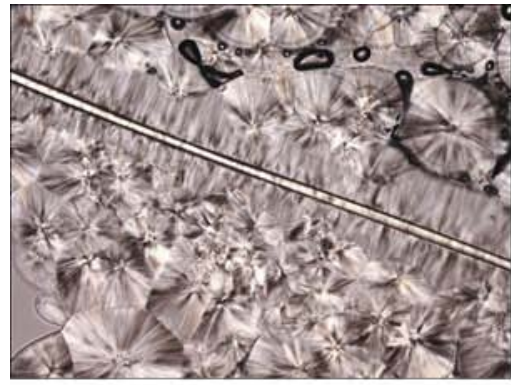

a)

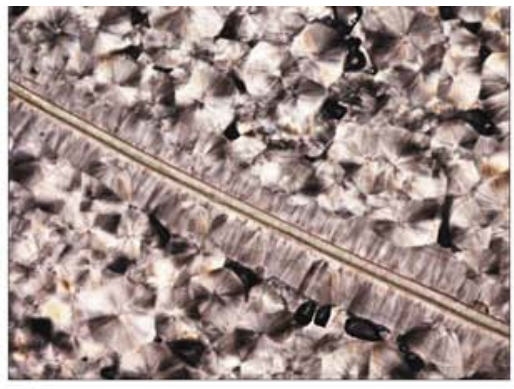

b)

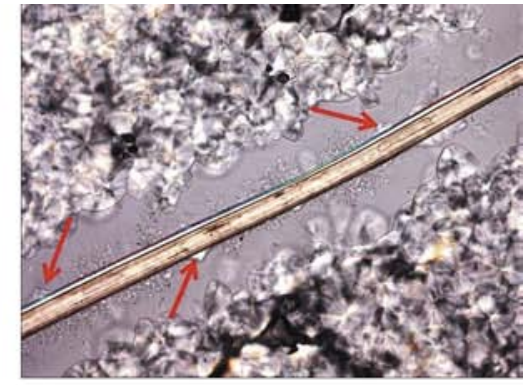

c)

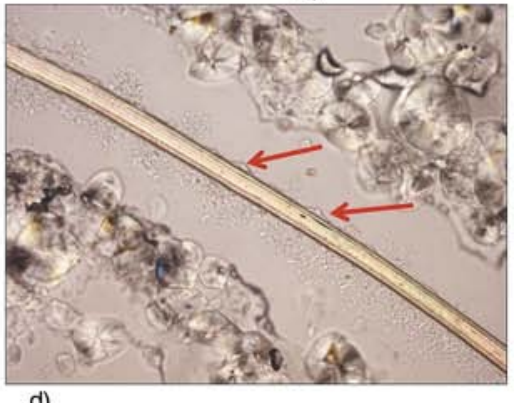

d)

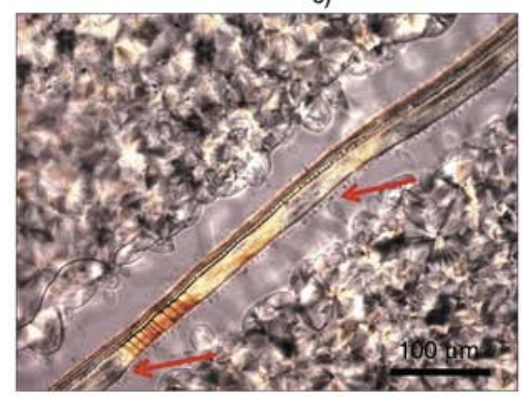

e)

Figure 3. The optical micrographs of the samples after selective melting at $158^{\circ} \mathrm{C}$ : (a) 0-PET/iPP; (b) 0.5-PET/iPP; (c) 1PET/iPP; (d) 1.5-PET/iPP; (e) 2-PET/iPP 
crystallities left behind by the separate melting of $\beta$ modification.

To further verify the crystalline form of the transcrystallinity, WAXD measurement was carried out. Because of a smaller amount of $\beta$-transcrystallinity existing in single fiber reinforced composites, $\beta$-transcrystallinity might not be easily detected by WAXD and DSC. Therefore, $5 \mathrm{wt} \%$ PET fibers were introduced into iPP matrix to fabricate abundant $\beta$-transcrystallinity which can be detected easily by DSC and WAXD. Figure 4 shows the WAXD result of the selected composites. Generally, for all samples, three apparent $\alpha$-crystal diffraction peaks appear at around $14.2,16.9$, and $18.7^{\circ}$, which correspond to $\alpha$ (110), $\alpha(040)$, and $\alpha$ (130), respectively. Furthermore, for 1-PET/iPP and 2-PET/iPP, it is obvious that there is an intense diffraction peak at approximately $16.1^{\circ}$, which corresponds to the $\beta(300)$ reflection of $\beta$-crystal [26]. It indicates that a certain amount of $\beta$-modification was generated in these composites. However, as the case of 0.5-PET/ iPP, there exists a very small $\beta$ (300) diffraction peak at $16.1^{\circ}$, indicating that an extremely small content of $\beta$-form crystal was formed in this composite. Furthermore, any evidence of $\beta$-modification can not be found in WAXD results of 0-PET/ iPP. Clearly, the WAXD result proves that $\beta$-NA loaded in the PET fibers can induce the formation of $\beta$-transcrystallinity. In addition, with the increasing content of $\beta$-NA loaded in PET fibers, the intensity of the reflection of $\beta(300)$ increases.

To gain deeper insight into the effect of $\beta$-NA loaded in PET fiber on non-isothermal crystallization of the composites, the crystallization and melting characteristics of the composites were studied by DSC (details of the sample preparation and

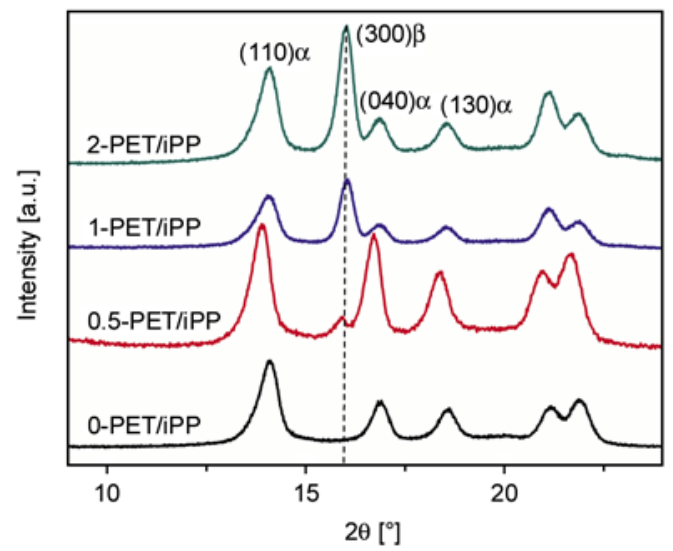

Figure 4. WAXD spectra of 0-PET/iPP, 0.5-PET/iPP, 1$\mathrm{PET} / \mathrm{iPP}$ and 2-PET/iPP composite samples

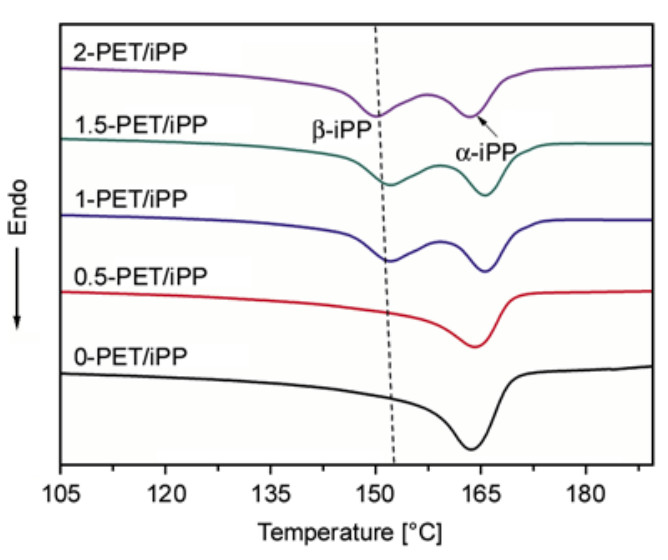

Figure 5. DSC melting curves of all the composites at a heating rate of $10^{\circ} \mathrm{C} / \mathrm{min}$

experimental procedures were described in Experimental Section). Figure 5 shows the DSC heating curves of all the samples. For the curves of 0-PET/ iPP and 0.5-PET/iPP, there is only one melting peak at $166.2^{\circ} \mathrm{C}$, revealing that $\alpha$-crystal is dominated in these composites. However, for 1-PET/iPP, 1.5-PET/ iPP and 2-PET/iPP, there exist double melting peaks, which are caused by different types of nucleation. The peak at the higher temperature (ca. $166.2^{\circ} \mathrm{C}$ ) is evidently $\alpha$-melting peak, and the other at the lower temperature $\left(\mathrm{ca} .152^{\circ} \mathrm{C}\right)$ is attributed to the melting of $\beta$-crystal. Once more, this result indicates that the $\beta$-NA in the PET fiber exhibits strong nucleating ability towards iPP matrix and leads to abundant $\beta$-modification, which is consistent well with the POM and WAXD results.

The non-isothermal crystallization curves are shown in Figure 6. One can observe that the onset crystallization temperature generally increases with the increasing content of $\beta$-NA loaded in PET fiber. For example, the onset temperature for 0 -PET/iPP is ca. $127^{\circ} \mathrm{C}$, and that for 0.5 -PET/iPP is a little higher

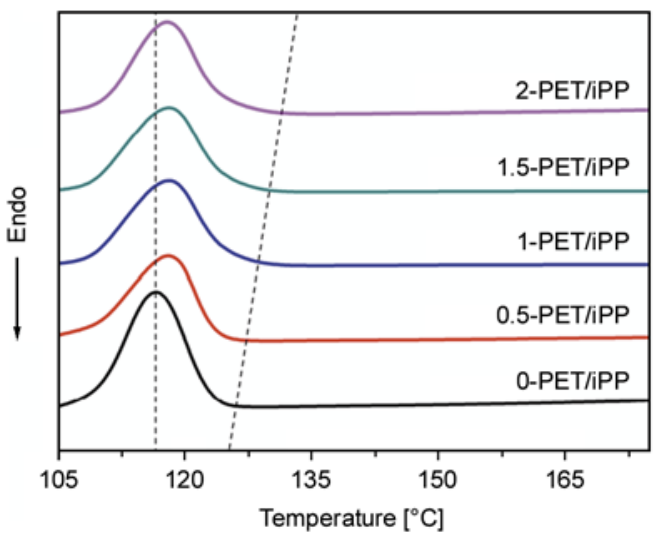

Figure 6. DSC cooling curves of all the composites at a cooling rate of $10^{\circ} \mathrm{C} / \mathrm{min}$ 
(ca. $128^{\circ} \mathrm{C}$ ). However, onset temperatures for 1-PET/ iPP, 1.5-PET/iPP and 2-PET/iPP are obviously above $130^{\circ} \mathrm{C}$. In addition, it can be clearly seen that the crystallization peak temperature for 0 -PET/iPP is ca. $116^{\circ} \mathrm{C}$, but that for the other four samples are ca. $118^{\circ} \mathrm{C}$. Clearly, the increased onset crystallization temperature and crystallization peak temperature are related to the elevating content of the $\beta-\mathrm{NA}$ filled in PET fiber.

\subsection{The mechanism for the formation of $\beta$-transcrystallinity in the presence of $\beta$-NA filled in PET fiber}

It is scientifically and technologically significant to understand the origin of $\beta$-transcrystallinity in the presence of $\beta$-NA loaded in PET fiber. From the above experimental results, it is evident that the occurrence of $\beta$-transcrystallinity is undoubtedly related to the $\beta$-NA loaded in the PET fiber. More importantly, there is a critical content of $\beta$-NA required (i.e., $1 \mathrm{wt} \%$ ) for the development of $\beta$-transcrystallinity. In other words, only the $\beta$-NA content exceeds this critical value, a certain amount of $\beta$-NA can be distributed on the surface of the PET fiber. Thus, the surface of the as-spun PET fibers with excessive $\beta$-NA content (take those containing 1

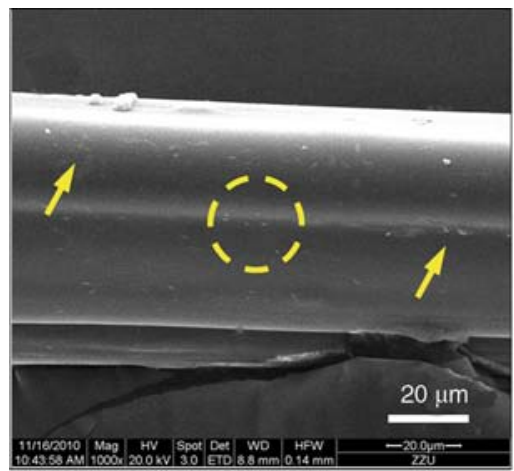

a)

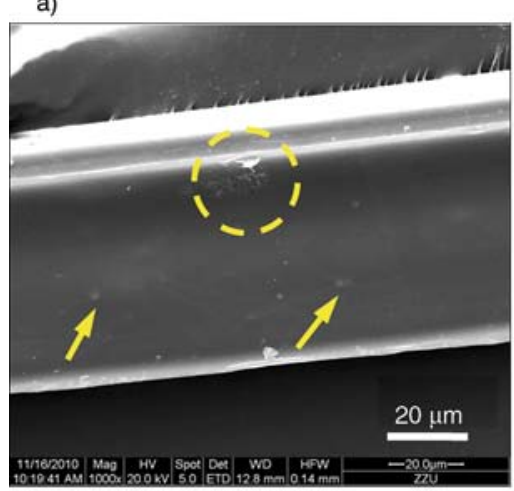

b) and $2 \mathrm{wt} \%$ as examples) were investigated by SEM, and the results are shown in Figure 7. In order to avoid the surface damage, no etching procedure was adopted. One observes that many aggregations of $\beta$-NA regularly distributed on the surface of the as-spun PET fiber containing $1 \mathrm{wt} \% \beta$-NA (shown by arrows in Figure 7a). Furthermore, as shown by Figure 7a', numerous aggregations with smaller size are also distributed on the surface of PET fiber (shown by arrows). For the case of the PET fiber containing $2 \mathrm{wt} \% \beta$-NA, although isolated aggregations of $\beta$-NA can be found on the surface of the asspun PET fiber (shown by the arrows in Figure 7b), much larger aggregation (shown by circle) can be also observed due to the excessive $\beta$-NA added. More interestingly, the larger aggregation consists of many oval-shaped entities with uniform size (shown by the arrows in Figure 7b'). The SEM result indicates that $\beta$-NA can distribute on the surface of PET fiber after being spun.

Note that this kind of $\beta$-NA has modest solubility at $200^{\circ} \mathrm{C}$, and can self-assemble into anisotropic entities during cooling [27]. Therefore, the aggregations in the region near PET fibers surface (shown in Figure 7) are a result of self-organization of partially molten $\beta$-NA during cooling process. In addi-

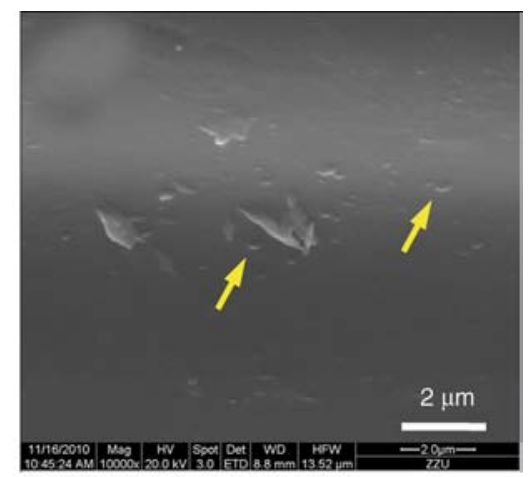

a')

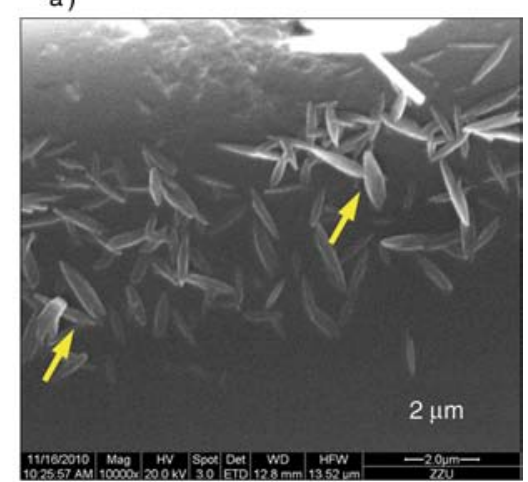

b')

Figure 7. SEM images showing the surface of as-spun PET fiber containing different contents of $\beta$-NA. (a) 1 wt $\%$; (b) $2 \mathrm{wt} \%$; (a') and (b') are enlarged images of the cricle area in (a) and (b), respectively. 


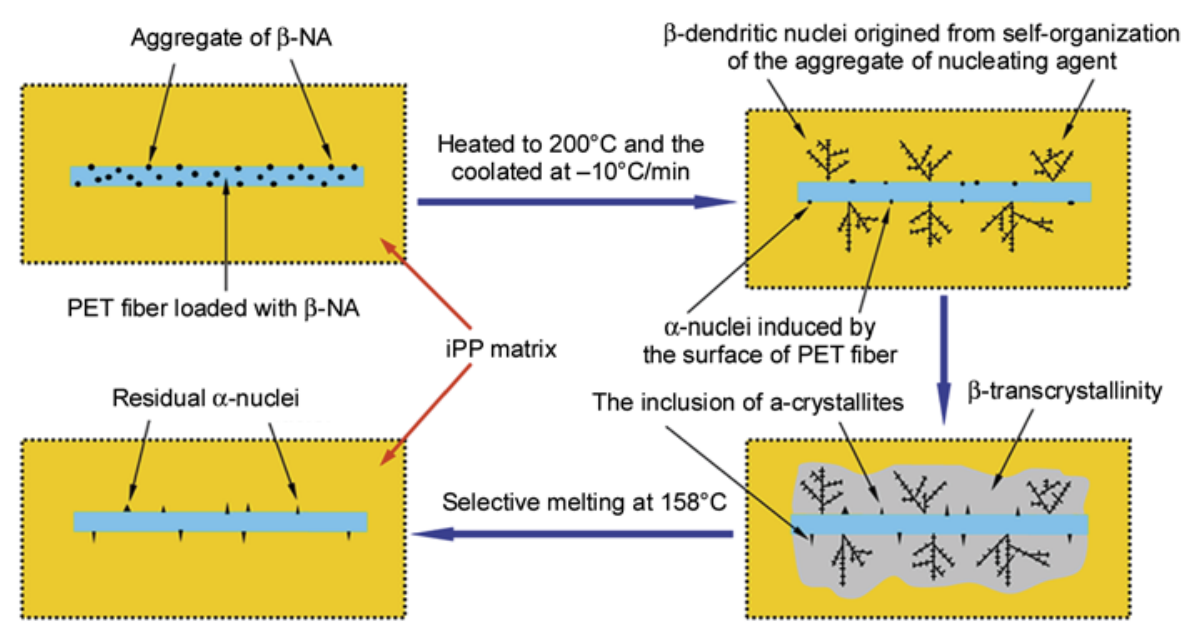

Figure 8. Schematic illustration of the interfacial morphology evolution of iPP reinforced by PET fiber loaded with $\beta$-NA and that after being selective melting

tion, during the process of self-organization, the $\beta$ NA loaded in PET fiber would go into the iPP component through such self-organization process and form dendritic structures [28]. Combined with the SEM images in this study, $\beta$-transcrystallinity can be understood as follows: during POM observation, when the composites were heated to $200^{\circ} \mathrm{C}$ and held for $5 \mathrm{~min}, \beta$-NA decorated on the PET fiber can be molten partially. During cooling, molten $\beta$ NA would self-assemble into a more complicated dendritic structures which nucleates the subsequent $\beta$-transcrystallinity into the branch-like agglomerate (Figure 2c, 2d and 2e) [9, 27].

Another interesting thing is that, after selective melting, many dot-like $\alpha$-nuclei appear on the surface of the fibers loaded with excessive $\beta$-NA (shown in Figure 3). It is well known that PET fiber can provide $\alpha$-nucleating sites for $\mathrm{PP}$ [16], which is also demonstrated in our study (see Figure 2a). Therefore, the PET fiber containing higher content of $\beta$-NA has dual nucleation ability, viz., it has both $\beta$ nucleating ability and $\alpha$ nucleating ability. It is logical that the $\beta$-NA loaded in the PET fiber would lead to dense $\beta$-nuclei and enhance the growth of $\beta$ transcrystallinity, while the local surface without $\beta$ NA of the fiber induce the formation of $\alpha$ crystallites. Accordingly, such interfacial morphology evolution and that after selective melting in samples containing larger amount of $\beta$-NA can be schematically shown in Figure 8.

\section{Conclusions}

The effect of $\beta$-NA loaded in the PET fibers on the crystal morphology at the interface and in the bulk was investigated by POM, DSC and WAXD. The result shows, for $0-\mathrm{PET} / \mathrm{iPP}$ and $0.5-\mathrm{PET} / \mathrm{iPP}$, the crystals in both iPP matrix and those at the interface are $\alpha$-phase. Furthermore, nuclei in the two areas appear at the same time. For 0.5-PET/iPP, though it has a small fraction of $\beta$-NA in the PET fiber, there is rarely $\beta$-NA on the lateral surface of the fiber, so it is hard to induce $\beta$-transcrystallinity. However, for 1-PET/iPP, 1.5-PET/iPP and 2-PET/iPP, the nuclei surrounding the fiber occur earlier than that in the matrix, indicating that $\beta$-NA loaded in the PET fiber shows heterogeneous nucleation towards iPP matrix. Furthermore, if the content of $\beta$-NA exceeds a critical value (i.e., $1 \mathrm{wt} \%$ ), the PET fiber loading $\beta$-NA shows nucleating duality. Meanwhile, the PET fiber loaded the content of $\beta$-NA above the critical value will show nucleating durability.

\section{Acknowledgements}

We would like to express our sincere thanks to NSFC (Grant: 50803060, 10872186, and 10872185) and the opening project of the key laboratory of polymer processing engineering, ministry of education of China.

\section{References}

[1] Tregub A., Harel H., Marom G., Migliaresi C.: The influence of thermal history on the mechanical properties of poly(ether ether ketone) matrix composite materials. Composites Science and Technology, 48, 185190 (1993).

DOI: 10.1016/0266-3538(93)90135-4 
[2] Yue C. Y., Cheung W. L.: Interfacial properties of fibrous composites. Part II Determination of interfacial shear strength, interfacial coefficient of friction, and the shrinkage pressure on the fibre. Journal of Materials Science, 27, 3181-3191 (1992).

DOI: $10.1007 / \mathrm{BF} 01116008$

[3] Nagae S., Otsuka Y., Nishida M., Shimizu T., Takeda T., Yumitori S.: Transcrystallization at glass fibre/ polypropylene interface and its effect on the improvement of mechanical properties of the composites. Journal of Materials Science Letters, 14, 1234-1236 (1995). DOI: $10.1007 / \mathrm{BF} 00291816$

[4] Saujanya C., Radhakrishnan S.: Development of a transcrystalline phase in poly(propylene) at the PET interface. Macromolecular Materials and Engineering, 286, 1-4 (2001).

DOI: $10.1002 / 1439-2054(20010101) 286: 1<1::$ AIDMAME1>3.0.CO;2-U

[5] Kitayama T., Utsumi S., Hamada H., Nishino T., Kikutani T., Ito H.: Interfacial properties of PP/PP composites. Journal of Applied Polymer Science, 88, 28752883 (2003).

DOI: 10.1002/app.11805

[6] Kotek J., Raab M., Baldrian J., Grellmann W.: The effect of specific $\beta$-nucleation on morphology and mechanical behavior of isotactic polypropylene. Journal of Applied Polymer Science, 85, 1174-1184 (2002). DOI: $10.1002 /$ app.10701

[7] Chen H. B., Karger-Kocsis J., Wu J. S., Varga J.: Fracture toughness of $\alpha$ - and $\beta$-phase polypropylene homopolymers and random- and block-copolymers. Polymer, 43, 6505-6514 (2002).

DOI: 10.1016/S0032-3861(02)00590-6

[8] Chvátalová L., Navrátilová J., Čermák R., Raab M., Obadal M.: Joint effects of molecular structure and processing history on specific nucleation of isotactic polypropylene. Macromolecules, 42, 7413-7417 (2009). DOI: $10.1021 / \mathrm{ma} 9005878$

[9] Varga J., Menyhárd A.: Effect of solubility and nucleating duality of $N, N^{\prime}$-dicyclohexyl-2,6-naphthalenedicarboxamide on the supermolecular structure of isotactic polypropylene. Macromolecules, 40, 2422-2431 (2007).

DOI: $10.1021 / \mathrm{ma} 062815 \mathrm{j}$

[10] Lovinger A. J., Chua J. O., Gryte C. C.: Studies on the $\alpha$ and $\beta$ forms of isotactic polypropylene by crystallization in a temperature gradient. Journal of Polymer Science: Polymer Physics Edition, 15, 641-656 (1977). DOI: $10.1002 /$ pol.1977.180150405

[11] Varga J.: $\beta$-modification of isotactic polypropylene: Preparation, structure, processing, properties, and application. Journal of Macromolecular Science Part B: Physics, 41, 1121-1171 (2002). DOI: $10.1081 / \mathrm{MB}-120013089$
[12] Varga J., Karger-Kocsis J.: Interfacial morphologies in carbon fibre-reinforced polypropylene microcomposites. Polymer, 36, 4877-4881 (1995).

DOI: 10.1016/0032-3861(95)99305-E

[13] Jay F., Haudin J. M., Monasse B.: Shear-induced crystallization of polypropylenes: Effect of molecular weight. Journal of Materials Science, 34, 2089-2102 (1999).

DOI: $10.1023 / \mathrm{A}: 1004563827491$

[14] Wang C., Liu C-R.: Transcrystallization of polypropylene composites: Nucleating ability of fibres. Polymer, 40, 289-298 (1999). DOI: 10.1016/S0032-3861(98)00240-7

[15] Sun X. L., Li H., Lieberwirth I., Yan S.: $\alpha$ and $\beta$ interfacial structures of the iPP/PET matrix/fiber systems. Macromolecules, 40, 8244-8249 (2007).

DOI: $10.1021 / \mathrm{ma} 071382 \mathrm{x}$

[16] Li H., Zhang X., Duan Y., Wang D., Li L., Yan S.: Influence of crystallization temperature on the morphologies of isotactic polypropylene single-polymer composite. Polymer, 45, 8059-8065 (2004).

DOI: 10.1016/j.polymer.2004.09.032

[17] Li H., Jiang S., Wang J., Wang D., Yan S.: Optical microscopic study on the morphologies of isotactic polypropylene induced by its homogeneity fibers. Macromolecules, 36, 2802-2807 (2003).

DOI: $10.1021 / \mathrm{ma} 034062 \mathrm{~W}$

[18] Wang S-W., Yang W., Bao R-Y., Wang B., Xie B-H., Yang M-B.: The enhanced nucleating ability of carbon nanotube-supported $\beta$-nucleating agent in isotactic polypropylene. Colloid and Polymer Science, 288, 681-688 (2010).

DOI: $10.1007 / \mathrm{s} 00396-010-2194-\mathrm{X}$

[19] Kawai T., Iijima R., Yamamoto Y., Kimura T.: Crystal orientation of $\beta$-phase isotactic polypropylene induced by magnetic orientation of $N, N^{\prime}$-dicyclohexyl-2,6naphthalenedicarboxamide. Polymer, 43, 7301-7306 (2002).

DOI: $10.1016 / \mathrm{S} 0032-3861(02) 00690-0$

[20] Khanna Y. P.: Rheological mechanism and overview of nucleated crystallization kinetics. Macromolecules, 26, 3639-3643 (1993). DOI: $10.1021 / \mathrm{ma} 00066 \mathrm{a} 024$

[21] Kotek J., Raab M., Baldrian J., Grellmann W.: The effect of specific $\beta$-nucleation on morphology and mechanical behavior of isotactic polypropylene. Journal of Applied Polymer Science, 85, 1174-1184 (2002). DOI: 10.1002/app.10701

[22] Dai K., Xu X-B., Li Z-M.: Electrically conductive carbon black (CB) filled in situ microfibrillar poly(ethylene terephthalate) (PET)/polyethylene (PE) composite with a selective CB distribution. Polymer, 48, 849-859 (2007).

DOI: $10.1016 /$ j.polymer.2006.12.026 
[23] Xiao W., Wu P., Feng J.: Effect of $\beta$-nucleating agents on crystallization and melting behavior of isotactic polypropylene. Journal of Applied Polymer Science, 108, 3370-3379 (2008).

DOI: $10.1002 /$ app. 27997

[24] Varga J.: $\beta$-modification of polypropylene and its twocomponent systems. Journal of Thermal Analysis and Calorimetry, 35, 1891-1912 (1989).

DOI: $10.1007 / \mathrm{BF} 01911675$

[25] Menyhárd A., Varga J.: The effect of compatibilizers on the crystallisation, melting and polymorphic composition of $\beta$-nucleated isotactic polypropylene and polyamide 6 blends. European Polymer Journal, 42, 3257-3268 (2006).

DOI: $10.1016 /$ j.eurpolymj.2006.09.003
[26] Turner Jones A., Aizlewood J. M., Beckett D. R.: Crystalline forms of isotactic polypropylene. Die Makromolekulare Chemie, 75, 134-158 (1964).

DOI: 10.1002/macp.1964.020750113

[27] Luo F., Geng C., Wang K., Deng H., Chen F., Fu Q., Na B.: New understanding in tuning toughness of $\beta$ polypropylene: The role of $\beta$-nucleated crystalline morphology. Macromolecules, 42, 9325-9331 (2009). DOI: $10.1021 / \mathrm{ma} 901651 \mathrm{f}$

[28] Varga J., Karger-Kocsis J.: The difference between transcrystallization and shear-induced cylindritic crystallization in fibre-reinforced polypropylene composites. Journal of Materials Science Letters, 13, 10691071 (1994).

DOI: $10.1007 / \mathrm{BF} 00277042$ 